\title{
COORDINATION BETWEEN THE RADIOASTRONOMY SERVICE AND THE RADIODETERMINATION SATELLITE SERVICE IN THE 1612 MHZ BAND
}

\author{
A. R. THOMPSON \\ National Radio Astronomy Observatory 1 \\ Charlottesville, Virginia 22903
}

\section{THE RADIODETERMINATION SATELLITE SERVICE}

The Radiodetermination Satellite Service (RDSS) provides radiolocation and limited communication for users of aeronautical, marine and terrestrial vehicles. A system of the RDSS is being developed in the United States by the Geostar Corporation (Rothblatt, 1988). In this system signals from a control center are transmitted to transceivers on users' vehicles through one of a series of two or three satellites spaced along the geostationary orbit, and responses are received through each of these satellites. If three satellites are used, locations of vehicles can be determined from the measured times of transmission from the control center to the user and back via different satellite paths. If two satellites are used the timing data must be supplemented by information such as altitude data from a terrain map, or an altimeter reading from the user's vehicle, which can be encoded in the response transmitted back. Computation of the vehicle location is performed at the control center. The equipment at the vehicle can be relatively simple since its basic function is that of a transponder, and intervention by the vehicle operator is not necessarily required. However, provision can also be made for the inclusion of short coded messages from the vehicle, so the unit on the vehicle is usually referred to as a transceiver. The system is designed to meet the needs of various users; for example, trucking companies which must periodically monitor the locations of vehicles in a widely distributed fleet. Position information can be relayed to the company headquarters as well as to the vehicle operator. The role played by the central control computer in determining the locations requires that all vehicles using the service identify themselves by a transmitted code. Thus developers and operators of RDSS systems can lease their services to vehicle users.

At the WARC (World Administrative Radio Conference) on the Mobile Services, Geneva, 1987, frequency bands were allocated to the RDSS, as follows:

$$
\begin{array}{ll}
\text { vehicle-to-satellite } & 1610.0-1626.5 \mathrm{MHz} \\
\text { satellite-to-vehicle } & 2483.5-2500.0 \mathrm{MHz} \\
& \text { and } 2500.0-2516.5 \mathrm{MHz}
\end{array}
$$

1. The National Radio Astronomy Observatory (N.R.A.O.) is operated by Associated Universities, Inc., under contract with the National Science Foundation. 
Links for communication between the satellites and the RDSS control center use frequencies within the allocations to the fixed satellite service. The signal of particular concern to radio astronomers is the uplink from vehicle to satellite which covers the secondary allocation to radio astronomy in the band 1610.6 - $1613.8 \mathrm{MHz}$. In radioastronomy, this band is used for observations of the $1612 \mathrm{MHz}$ hydroxyl line. Coordination between the RDSS and the radioastronomy service in this band is the main subject of this paper.

There is also a possibility of interference from the second harmonic of the $2483.5-2500.0 \mathrm{MHz}$ downlink signal to radioastronomy observations in the 4800 - $4990 \mathrm{MHz}$ (secondary) and $4990-5000 \mathrm{MHz}$ (primary) radioastronomy bands. The expected spectral power flux density of the downlink signal at the earth's surface is $-181 \mathrm{~dB} \mathrm{~W} \mathrm{~m}^{-2} \mathrm{~Hz}^{-1}$ (CCIR Report 1050). The harmful threshold of interference in these radio astronomy bands for a signal from a satellite in geostationary orbit is $-256 \mathrm{~dB} \mathrm{~W} \mathrm{~m}^{-2} \mathrm{~Hz}^{-1}$ (CCIR Report 224-6). Thus the second harmonic radiation from the RDSS satellite must be $75 \mathrm{~dB}$ below the fundamental to avoid interference.

\section{COORDINATION BETWEEN THE RDSS AND RADIOASTRONOMY}

As a means of reducing interference to radioastronomy from RDSS vehicleto-satellite transmissions, the system under development in the United States includes a procedure in which transmissions from user transceivers within specified zones surrounding radioastronomy observatories occur only during the first $200 \mathrm{~ms}$ of each second of Coordinated Universal Time (UTC) (Rothblatt, $1987,1988)$. The scheme is put into effect during periods for which an observatory has notified the RDSS system operator that observations in the $1610.6-1613.8 \mathrm{MHz}$ band are to be made. The radioastronomy receivers at the observatory are blanked during these $200 \mathrm{~ms}$ intervals, and the resulting $20 \%$ loss in observing time results in an overall loss in sensitivity of $10 \%$.

The control of the vehicle transmissions is achieved through the timing sequence established at the RDSS control center, which consists of a series of time frames approximately $12 \mathrm{~ms}$ long. Signals defining the time frames are transmitted from the central station via the satellites. Transmissions from users are triggered by time marks within the frames. Users within a radioastronomy protection zone will have a flag set in the control logic of their transceivers so that they can be triggered only in time frames in which the signal from the control center contains a special enabling code. Such time frames will be chosen so that user transmissions fall within the $200 \mathrm{~ms}$ interval described above. The flag in the user's transceiver will be set or reset by transmissions from the RDSS control center. Transmissions from vehicles within a radioastronomy zone, but outside the $200 \mathrm{~ms}$ intervals, may occur when a user enters a radioastronomy zone with the vehicle's RDSS transceiver switched off, and then switches it on before leaving the zone. Such occurrences can be eliminated if, whenever a transceiver is switched on, the flag is initially set for transmission in the $200 \mathrm{~ms}$ intervals only.

The transmitter output power of an RDSS transceiver is approximately 40 W (CCIR Report 1050), and the gain of the transmitting antenna in a horizontal direction is close to $0 \mathrm{dBi}$. Thus the power level received in a radiotelescope through sidelobes of gain $0 \mathrm{dBi}$ from a transceiver at a distance of $100 \mathrm{~m}$ is less 
than $10^{-6} \mathrm{~W}$. So long as a distance of at least $100 \mathrm{~m}$ is maintained between a radiotelescope and any RDSS user, low-noise input stages of gain 20-30 dB in the radiotelescope should not be overloaded. If a diode switch to reject input signals during the $200 \mathrm{~ms}$ intervals is placed following such input stages, no significant increase in receiver noise will result from the insertion loss of the switch. There should therefore be no technical problems in blanking of the radiotelescope during the $200 \mathrm{~ms}$ intervals.

\section{RADIOASTRONOMY PROTECTION ZONES}

\subsection{General Considerations}

The feasibility of the proposed time sharing between radioastronomy and the RDSS is determined mainly by the possibility of defining zones that are large enough to provide effective protection for radioastronomy, without causing an unacceptable restriction to the radiodetermination service. Article 11 of the Radio Regulations indicates that in international coordination of assignments to earth stations in the radiodetermination satellite service, the distances over which effects on services should be considered extend to $100 \mathrm{~km}$ from ground-based RDSS earth stations, and to $400 \mathrm{~km}$ from airborne earth stations. These regulations apply to interference between RDSS users in one country and services in another country. For coordination within any individual country, the size of the protection zones will be determined by the administration concerned. In the United States of America, the radioastronomy protection zones are currently defined as having radii $25 \mathrm{~km}$ for ground-based RDSS users and $150 \mathrm{~km}$ for airborne users.

The transmission from any user takes place in a series of $12 \mathrm{~ms}$ time frames, and for each position determination is expected to last no more than about $0.1 \mathrm{~s}$. For any single user such a transmission will occur, perhaps, about once per hour for a terrestrial vehicle. In that case the average power radiated by the user is about $46 \mathrm{~dB}$ below the level during a transmission. Note that it is the average power within a time interval determined by the type of observation, rather than the peak power, that is important with regard to interference thresholds, provided that the peaks do not cause non-linear responses in the radioastronomy system. The total signal level will depend upon the number of users, and will vary with time in a quasi-random manner. The principal uncertainty in calculating the required sizes of radioastronomy protection zones is the number and distribution of RDSS users when the system becomes fully developed. The Geostar system uses 8 beams to cover the continental U.S., each beam being approximately $2.5^{\circ}$ wide and having a footprint of width $1600 \mathrm{~km}$ and area $2.0 \times 10^{6} \mathrm{~km}^{2}$. Coding of the transmissions allows for reception of up to 32 simultaneous signals from users within the footprint of each beam, and this represents the peak capacity. The planned average capacity is approximately 10 simultaneous users per beam (CCIR Report 1050).

For a transceiver output power of $40 \mathrm{~W}$, and a gain of $0 \mathrm{dBi}$ in the horizontal direction of the transmitting antenna, the total isotropically radiated power that contributes to the signal level at an observatory is, on average, $400 \mathrm{~W}$ per beam footprint area, or $2 \times 10^{-4} \mathrm{~W}$ in each square kilometer. The data transmission uses spread spectrum modulation with a chip rate of $8.192 \mathrm{MHz}$. This radiated 
spectrum has a central lobe of width $16.384 \mathrm{MHz}$ between first nulls, and the spectral power flux density at the band center is equal to that for a rectangular spectrum of width $8.19 \mathrm{MHz}$. The power density of $2 \times 10^{-4} \mathrm{~W} \mathrm{~km}^{-2}$ thus corresponds to a spectral power density of $2.4 \times 10^{-11} \mathrm{~W} \mathrm{~Hz}^{-1}$ per square kilometer at $1618.25 \mathrm{MHz}$, which is the peak of the transmitted spectrum. At the nearest edge of the radioastronomy band, $1613.8 \mathrm{MHz}$, the spectral power density is $5 \mathrm{~dB}$ lower, and this level will be used in calculation of interference to spectral line observations. The mean level over the radioastronomy band, which is $11 \mathrm{~dB}$ lower than that at the peak of the spectrum, will be used for continuum observations.

\subsection{Protection Zones for Transceivers on Terrestrial Vehicles}

Consider a radio telescope at the center of a circular zone, within which transmissions occur only when the radio telescope receiver is blanked. The radius of the zone must be chosen so that the cumulative effect of transmissions outside of it does not exceed the harmful threshold for the type of radio telescope at the center. Since the signals are broadband, we specify the harmful threshold in terms of spectral power flux density. Values for five types of radioastronomy observations are shown in column 2 of Table I. The total power values are equivalent to those in Tables I and II of CCIR Report 224-6, except that they have been adjusted for more appropriate receiver bandwidths. These are $1 \mathrm{kHz}$ for spectral line observations of $\mathrm{OH}$ at $1612 \mathrm{MHz}$, and $3 \mathrm{MHz}$, equal to the width of the (secondary) allocated band, for continuum observations. For synthesis arrays and very long baseline interferometry (VLBI) the harmful thresholds are adapted from Thompson, Moran, and Swenson (1986). For very long baseline interferometry (VLBI) the thresholds are the same for both the spectral line and continuum cases, and the spectral power density levels at $1613.8 \mathrm{MHz}$ will be used. Note that the $1612 \mathrm{MHz}$ radio astronomy band is used almost exclusively for spectral line observations, but calculations for continuum observations have been included here for completeness.

In calculations of the interference from terrestrial vehicles, the total spectral power density values discussed in Section 3.1 will be used, since other users are expected to be only a minor fraction of the total. Values for the radius of the coordination zone that result in signal levels equal to the thresholds in column 2 of Table I are given in column 3. These were computed from the propagation curves given by Rice et al. (1967) for levels not exceeded for more than $10 \%$ of time. These curves include the effects of tropospheric scatter as well as diffraction around a smooth spherical earth. The published curves apply for antenna heights of $30 \mathrm{~m}$, and results were corrected to ground level using the height gain data in CCIR Report 715. The integration over distance was approximated by summing contributions from consecutive annular rings of width $10 \mathrm{~km}$. No allowance was made for the height of the radio telescope or for propagation through trees: in practice these two effects would roughly cancel one another. Clearly the values in column 3 of Table 1 indicate that for a smooth earth model the radius of the coordination zone should be much greater than 25 $\mathrm{km}$. However, many observatory sites are chosen to provide some degree of shielding by surrounding terrain. Columns 4 and 6 show the height of a single mountain ridge surrounding the observatory at a distance of $10 \mathrm{~km}$ or $20 \mathrm{~km}$ for which diffraction would result in signal levels equal to the harmful thresholds for 
a radioastronomy zone of radius $25 \mathrm{~km}$. Computational methods in CCIR Report 712 were followed, and the mountain ridge was assumed to have a radius of $200 \mathrm{~m}$ at the top. The four-thirds earth radius approximation was used to take account of atmospheric refraction here and in all calculations of diffraction and horizon distances in this report. Contributions from the annular zones were included out as far as the horizon as seen from the mountain top. For distances beyond this horizon, the path loss increases rapidly. Columns 5 and 7 give the elevation angle of the mountain top subtended at the observatory. Note that it is the elevation angle rather than the actual height of the mountain that is important, since the propagation loss depends upon the angle of bending of the radio path. Propagation by tropospheric scatter should not cause problems so long as the horizon elevation at the transmitter or receiver is about $1^{\circ}$ or greater.

From Table I it can be concluded that for a $25-\mathrm{km}$ radius of the radioastronomy protection zone, shielding by mountains of elevation angle up to $3^{\circ}-4^{\circ}$ is required for spectral line observations, and up to $6^{\circ}-7^{\circ}$ for continuum observations. The Dominion Radio Astrophysical Observatory at Penticton, Canada, is an example of an exceptionally well shielded site, with a horizon elevation greater than $5^{\circ}$ in almost all directions. A more representative example of what may be considered well shielded is the facility of the U.S. National Radio Astronomy Observatory (NRAO) at Green Bank, West Virginia. At most positions within the observatory grounds the horizon angle is at least $2-1 / 2^{\circ}$ for more than $330^{\circ}$ of azimuth, and at some positions it is in the range $3-1 / 2^{\circ}$ to 4 $1 / 2 \circ$ for all azimuths. In contrast, many radio observatories have very little mountain shielding. This is particularly true for sites of large antenna arrays which require extensive flat areas. For example, at the NRAO Very Large Array (VLA) site in New Mexico, the mountains that define the horizon are largely at distances of $20 \mathrm{~km}$ and greater, and subtend angles of only $1^{\circ}$ or $2^{\circ}$ over limited azimuth ranges. Array sites with even less protection by mountains include the site of the Westerbork Synthesis Radio telescope in the Netherlands and the Australia Telescope at Culgoora, Australia.

\subsection{Protection Zones for Transceivers on Aircraft}

The number of aircraft which may be fitted with RDSS transceivers is expected to be much less than the number of terrestrial vehicles. Here it will be assumed that an average of one in twenty-five of all transceiver transmissions are radiated from aircraft. This would correspond to, say, one airborne user for every 100 ground-based users, but obtaining locations about four times more often. Note that it is assumed that RDSS systems on aircraft will mostly be used for navigation on long flights, and are not likely to replace existing systems in the proximity of airports and other situations where rapid updating of positions is required. The mean spectral power density for airborne RDSS transcejvers corresponding to the transmission rate assumed above is $9.6 \times 10^{-13} \mathrm{~W} \mathrm{~Hz}^{-1}$ per square kilometer at the peak of the transmitted spectrum. At a distance of 150 $\mathrm{km}$, any aircraft above $1300 \mathrm{~m}$ altitude would be within line of sight of the observatory for a smooth-earth model. An aircraft at $10,000 \mathrm{~m}$ would be within line of sight for distances up to $410 \mathrm{~km}$. Thus, unless the radioastronomy zones are as large as $400 \mathrm{~km}$ in radius, or the aircraft heights are no greater than 1,000$2,000 \mathrm{~m}$, interference can only be avoided for observatories shielded by mountains. 
Table II gives the heights and elevation angles of mountains required to meet the harmful thresholds of Table I for aircraft heights of $1,000,5,000$, and $10,000 \mathrm{~m}$. Again these are based on diffraction effects, and methods in CCIR Report 712 were used. For columns 2-7, the signal levels at the observatory were calculated for annular rings of width $50 \mathrm{~km}$ and summed for radii from $150 \mathrm{~km}$ out to the distance for which the aircraft would be on the horizon as seen from the mountain top. Except for the unusual case of continuum observations in this band, mountain elevations of $2.5^{\circ}-3^{\circ}$ appear to provide adequate shielding for aircraft heights up to about $5,000 \mathrm{~m}$, which is above the commonly used altitudes for small, unpressurized aircraft. For large aircraft flying at $10,000 \mathrm{~m}$ and above, greater elevation angles are necessary. Columns 8 and 9 of Table II show results for similar calculations for a radioastronomy protection zone of radius $400 \mathrm{~km}$. In this case only relatively low elevation angles are required to provide shielding. Propagation by tropospheric scatter should only be important in the cases in Table II for which the horizon elevation is less than about $1^{\circ}$, and may set the lower limit on the required elevation in these cases.

\section{DISCUSSION}

Two features of the RDSS system currently being implemented distinguish it from most other radiolocation or communication systems and allow the possibility of coordination with radioastronomy observatories. These are the knowledge of the positions of the users at the central control station, and the control of the timing of the transmissions of the user transceivers by the central control system. Thus coordination of the RDSS and radioastronomy services is possible using geographical and timing parameters to separate the signals.

The results in Tables I and II are based on a number of simplifying assumptions. The uniform area density of user transmitters is clearly an oversimplification, but provides a basis for a general analysis rather than one for a particular radioastronomy observatory site. In practice the density of users will vary significantly reaching peaks at points where major highways converge and near cities and airports. The single mountain range surrounding the observatory is also a simplification, since in real situations multiple peaks or ridges between the radio telescope and the transmitter may introduce further loss. Also, the fraction of the total transmissions emanating from transceivers on aircraft when the system is fully developed can only be roughly estimated. However, the conclusion can be drawn that for a smooth terrain without mountains, radioastronomy protection zones of radius $50-100 \mathrm{~km}$ are required for terrestrial users and about $400 \mathrm{~km}$ for airborne ones. With much smaller zones, interference to radioastronomy can only be avoided at observatory sites that are heavily shielded by surrounding mountains. It is important to note that such levels of shielding are mainly found in mountain valleys of dimensions that limit the radio telescopes to single antennas or compact arrays. In the case of the VLA, for example, the antennas are spread over an area approximately $40 \mathrm{~km}$ in diameter, and a high plains area was chosen to accommodate the array. For transceivers on high altitude aircraft, the protection zones required to avoid lineof-sight propagation to observatories with little or no mountain shielding may include a large fraction of the aircraft flight paths. If, however, such aircraft are a small fraction of the total users, and account for only a few percent of the total 
transmissions, it should be possible to accommodate them within the $200 \mathrm{~ms}$ coordination intervals.

The planning of coordination zones should ideally be done on an individual site basis, and should include an appraisal of the terrestrial features and distribution of RDSS users within several hundred kilometers of each observatory, as well as the types of observations made there. The results in Tables I and II provide some initial estimates of the probable requirements.

\section{TABLE I}

Protection of radio astronomy observatories from ground-based RDSS transmissions for five types of observations. These are spectral line and continuum observations with both total power radio telescopes and synthesis arrays, and observations using very long baseline interferometry. Column 3 gives the radius of the required protection zone for a smooth earth model. Columns 47 give the heights and elevations of mountains at distances of 10 and $20 \mathrm{~km}$ that would provide the required protection for a zone radius of $25 \mathrm{~km}$.

\begin{tabular}{|c|c|c|c|c|c|c|}
\hline $\begin{array}{l}\text { Type of } \mathrm{Ha} \\
\text { Observation Thr } \\
\text { (dB W }\end{array}$ & $\begin{array}{l}\text { emful } \\
\text { eshold } \\
n^{-2} \mathrm{~Hz}^{-1} \text { ) }\end{array}$ & $\begin{array}{l}\text { Radius of } \\
\text { Coordination } \\
\text { Zone } \\
(\mathrm{km})\end{array}$ & $\begin{array}{l}\text { Sh } \\
\text { Dist } \\
\text { Hgt } \\
\text { (m) }\end{array}$ & $\begin{array}{l}\text { elding } \\
10 \mathrm{~km} \\
\text { Elev. } \\
\text { Angle }\end{array}$ & $\begin{array}{l}\text { Moun } \\
20 \\
\text { Hgt } \\
(\mathrm{m})\end{array}$ & $\begin{array}{l}\text { ain } \\
\mathrm{km} \\
\text { Elev. } \\
\text { Angle }\end{array}$ \\
\hline $\begin{array}{l}\text { Total Power, } \\
\text { Spectral Line }\end{array}$ & -230 & 81 & 720 & $4.1^{\circ}$ & 1120 & $3.2^{\circ}$ \\
\hline $\begin{array}{c}\text { Total Power, } \\
\text { Continuum }\end{array}$ & -250 & $>100$ & 1220 & 7.0 & 2020 & 5.8 \\
\hline $\begin{array}{c}\text { Synthesis Array } \\
\text { spectral Line }\end{array}$ & -215 & 49 & 300 & 1.7 & 420 & 1.2 \\
\hline $\begin{array}{l}\text { Synthesis Array } \\
\text { Continuum }\end{array}$ & -232 & 69 & 600 & 3.4 & 900 & 2.6 \\
\hline VLBI & -209 & 40 & 180 & 1.0 & 240 & 0.7 \\
\hline
\end{tabular}




\section{TABLE II}

Heights and elevations angles of mountains at a distance of $10 \mathrm{~km}$ from an observatory required to provide shielding from airborne RDSS systems. For a radius of $150 \mathrm{~km}$ for the radio astronomy protection zone, three aircraft heights -$1,000 \mathrm{~m}, 5,000 \mathrm{~m}$, and $10,000 \mathrm{~m}$-- are considered. For a zone radius of $400 \mathrm{~km}$, shielding by mountains is required only for the greatest of these three heights.

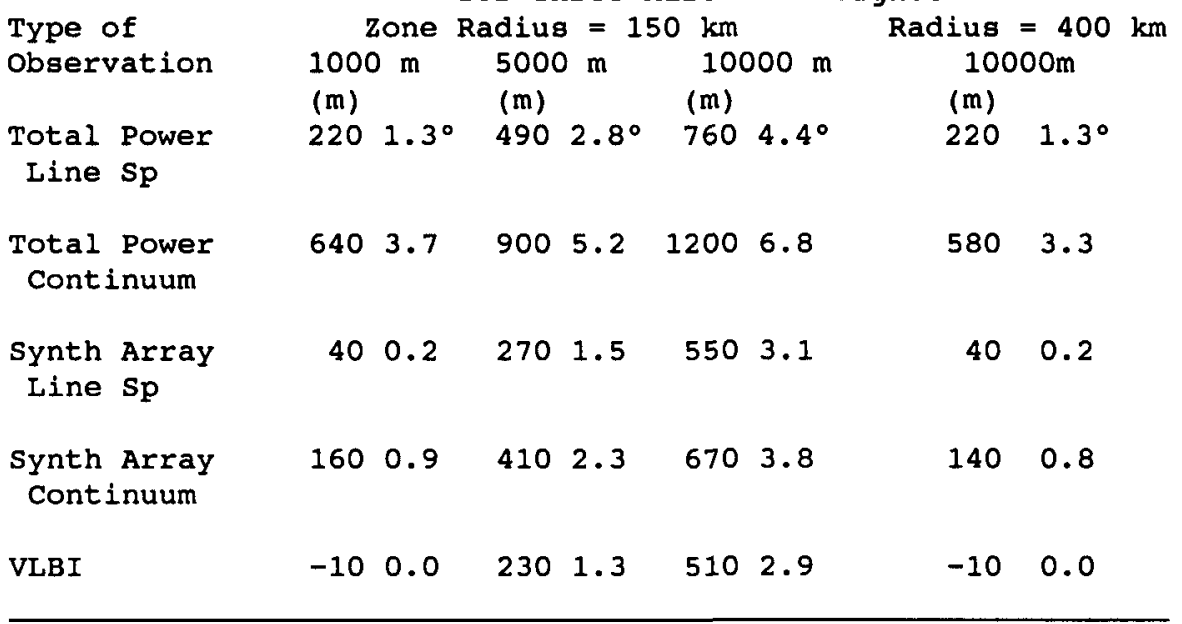

\section{REFERENCES}

Recommendations and Reports of the CCIR (International Radio Consultative Committee), published by the International Telecommunication Union, Geneva, 1986.

Rice, P. L., Longley, A. G., Norton, K. A., and Barsis, A. P. (1967), National Bureau of Standards Technical Note 101, Revisions I and II, AD687820 and AD687821, National Technical Information Service, Springfield, Virginia, USA

Rothblatt, M. A. (1987), Radiodetermination Satellite Services and Standards, Artech House Inc., Norwood, Massachusetts, USA.

Rothblatt, M. A. (1988), "How Geostar Radiodetermination Technology Minimizes Interference to Radioastronomy," Proceedings of LAU Colloquium No. 112, (this volume).

Thompson, A. R., Moran, J. M. and Swenson, G. W., Jr. (1986), Interferometry and Synthesis in Radio Astronomy, John Wiley and Sons, New York: see Ch. 14. 